\title{
Photographies et mémoires de la Première Guerre dans les guides Michelin (1917-2019)
}

\section{Françoise Knopper}

Université Toulouse Jean Jaurès

\section{Abstract}

Die Guides Illustrés des Champs de bataille von Michelin sind keine gewöhnlichen Reiseführer. Sie sammelten und veröffentlichten von 1917 bis 2019 eine beträchtliche Anzahl von Fotografien. Die Wiederverwendung und Hinzufügung von Abbildungen zeugen von der Verflechtung des kommunikativen Gedächtnisses und des kulturellen Gedächtnisses. Bis 1921 werden die Abbildungen dem Klima des Kriegsendes gerecht und der militärische Fotojournalismus hat ein Monopol: Ereignisse und materielle Realität der Zerstörung herrschen vor. In der Zwischenkriegszeit blieben dieses Monopol und das objektivierende Wissen über den Ersten Weltkrieg bestehen und wurden komplexer, da sie sich auf Verdun als einen konkreten Bezugspunkt, aber auch als einen entstehenden Mythos konzentrierten. Der Reiseführer von 2019 wechselt die Paradigmen und nimmt Friedhöfe, Kriegsdenkmäler und Stelen als Ausgangspunkt, um das Interesse aller und das Einfühlungsvermögen jedes Besuchers in Einklang zu bringen, der Vergangenheit zu gedenken und sie in Zukunft symbolisch zu bewahren.

Les Guides Illustrés des Champs de bataille de Michelin ne sont pas des guides touristiques habituels. Ils ont collecté et publié un nombre considérable de photogravures entre 1917 et 2019. Réemploi et ajouts de gravures font constater l'intrication de la mémoire communicative et de la mémoire culturelle. Jusqu'en 1921, les illustrations montrent le climat de la sortie de guerre et le photojournalisme militaire a le monopole : l'événement et la réalité matérielle de la destruction s'imposent. Durant l'entre-deux-guerres, ce monopole et la connaissance objectivante de la Première Guerre perdurent et se complexifient puisqu'ils se concentrent sur Verdun, à la fois référent concret et mythe émergent. Le guide de 2019 change les paradigmes et prend les cimetières, monuments aux morts, stèles, comme point de départ pour concilier l'intérêt de tous et l'empathie de chaque visiteur, pour commémorer le passé et pour le préserver symboliquement dans le futur.

Michelin's Illustrated Battlefields Guides are no ordinary travel guides. A considerable number of photo-engravings were collected and published between 1917 and 2019. Reuse and addition of engravings demonstrate the interdependence of communicative memory and cultural memory. Up to 1921 the illustrations bore witness to the end of war / post-war climate, and military photojournalism still enjoyed a monopoly: events and material reality of destruction prevailed. During the interwar period, this monopoly and the objectifying knowledge of World War I persisted and became more complex, focusing on Verdun both as a concrete referent and an emerging myth. The 2019 guide changes paradigms and takes cemeteries, war memorials, stelae, as a starting point, with a view to reconciling the interest of all and the empathy of each visitor, commemorating the past and symbolically preserving it for the future.

\section{Keywords}

Michelin • Reiseführer • Erster Weltkrieg • Trümmern • kulturelles Gedächtnis

Michelin $\bullet$ guide de voyage $\cdot$ Première Guerre $\cdot$ ruines $\bullet$ mémoire culturelle

Michelin $\bullet$ travel Guide $\bullet$ World War I ruins $\bullet$ cultural memory

Les premières maisons de Reims nous la cachent. Nous arrivons au parvis.

Ce n'est plus elle, ce n'est que son apparence.

C'est un soldat que l'on aurait jugé de loin sur sa silhouette toujours haute, mais qui, une fois approché, ouvrant sa capote, vous montrerait sa poitrine déchirée.

Les pierres se détachent d'elle. Une maladie la désagrège. Une horrible main l'a écorchée vive.
Les photographies ne vous diront pas son état. Les photographies ne donnent pas le teint du mort. Vous ne pourrez réellement pleurer que devant elle, quand vous y viendrez en pèlerinage.

(...) La cathédrale de Reims n'est plus qu'une plaie.

(Albert Londres, "L'agonie de la basilique », article paru dans

Le Matin », 29-09-1914). 
L'entreprise Michelin, outre la production industrielle qu'elle a assurée pendant la Première Guerre (Moulin-Bourret 1997, laventure.michelin 2017), a publié en 1917, en accord avec la propagande (Krumeich 1994), les deux premiers de ses Guides Illustrés des Champs de bataille. Puis 29 Guides sont sortis entre 1919 et 1921 , chacun se concentrant sur une ville et sur les champs de bataille alentour de manière à couvrir tous les hauts-lieux des combats ; les couvertures en étaient sobres, minimalistes, brochées ou avec une couverture bleue en moleskine. Une deuxième phase se situe entre 1925 et 1937 : Verdun Argonne est alors augmenté et plusieurs fois réédité ; 1929, année riche en parutions commémorant la Guerre et dues à des militaires allemands et français ou à des défenseurs notoires de positions pacifistes, voit paraître sa seule traduction en allemand. En un troisième temps, Michelin, tenant compte des technologies numériques, a lancé une campagne de rééditions à partir de 2012. Et, en dernier lieu, Champs de bataille de la Première Guerre mondiale en France et en Belgique a paru en 2019 : tout à la fois guide synthétique et catalogue doté d'illustrations en couleurs, il offre une rétrospective thématique, recommande des sites et attribue - parcimonieusement - quelques étoiles. L'emploi du pluriel Champs dans l'intitulé de cette collection relève des ouvrages ayant l'intention de " donner sens au conflit » à partir « de larges corpus et de larges espaces » (Loez/Offenstadt : 2013, 193), en l'occurrence avec une abondante iconographie montrant monuments, paysages, chefs militaires, groupes de soldats, et circonscrite aux lieux situés en France et en Belgique.

Ces guides Michelin ont collecté et publié un nombre considérable de photogravures, davantage même que d'autres guides parus dans les années 1917-1921. C'est d'abord la conséquence du plan thématique appliqué dans ces ouvrages : origine du lieu et principales phases de son passé, puis faits militaires de $14-18$ et pour finir circuit touristique préconisé. Les scènes de villes ou de campagnes photographiées en temps de guerre et après-guerre alternent avec des clichés qui remontent à avant 1914 et cette narration en images replace chaque lieu dans son continuum. Prises dans leur globalité, les illustrations confèrent une cohérence sérielle propre à chacune des phases éditoriales de ces guides, mais il y a des interrogations pérennes auxquelles ils pouvaient apporter des éléments de réponse : faut-il ou non reconstruire ce qui a été détruit ? quel est le rôle de l'esthétique dans un guide sur les champs de bataille ? le message à transmettre aux futurs visiteurs est-il national ou transnational ?

La spécificité de ces illustrations réside en effet moins dans les motifs retenus - car bien d'autres supports montrent monuments endommagés et paysages dévastés (Paul 2009 ; Becker 2014 ; Gaehtgens 2018 : 54-134) - que dans le fait qu'elles étaient archivées pour des touristes et que leur esthétique est fonctionnelle : ce sont des aide-mémoires pour signaler ce que l'on peut ou doit voir à l'étape recommandée. Ce faisant, ces guides jouent un rôle performatif : en tant que réceptacles d'images, ils stimulent la mémoire visuelle et préparent le passage de la mémoire communicative - celle des témoins - à la mémoire culturelle - celle qui reste gravée dans le marbre ; ils servent de relais patrimonial (Jalabert / Puton 2014). Or, la réutilisation que, d'une phase à l'autre, les guides Michelin font de leurs illustrations initiales met en évidence le glissement d'une mémoire à la suivante ainsi que l'intrication des deux types de mémoire - sur laquelle les chercheurs insistent d'ailleurs dorénavant (Düllo 2011, 192).

Étant donné cette profusion et ces réemplois, les illustrations gagnent à être étudiées en tant que "documents " au sens que Foucault donne à ce terme : elles sont certes des « instruments » utiles en ce qu'elles aident à connaître le passé mais elles possèdent aussi une signification intrinsèque, celle de la représentation que ces divers guides Michelin proposent de la Première Guerre. Surtout à partir des vues non événementielles, monuments, routes et rues, paysages, qui composent dans chacun d'eux un discours cohérent, nous examinerons les modalités de la linéarité tout comme de la discontinuité dans leur présentation des cicatrices de la Guerre.

\section{TOURISME DE GUERRE : PÈLERINAGE, MÉMOIRE ET HISTOIRE}

Le tourisme dit « de guerre » résultait de trois traditions : celle du pèlerinage et de sa vocation morale; celle de l'inspection de professionnels militaires puisque la visite de champs de bataille était prévue dans la formation des officiers qui arpentent les lieux pour analyser la stratégie; enfin celle de l'industrie du tourisme.

En termes de culture, I'historiographie française et allemande récente (Beaupré, Brandt, Harp, Hertzog, Heymel, Jalabert, Prost, Riederer e.a.) conclut à une évolution des fonctions du tourisme sur les champs de bataille au fil du $X X^{e}$ siècle : le « tourisme de pèlerinage " a cédé la place au " tourisme d'histoire » dans les années 1960, lequel a été ensuite relégué au second plan par le " tourisme de mémoire ». Les guides Michelin n'échappent pas à cette évolution mais l'éclairage qu'y donne le paratexte (avant-propos, légendes) et l'utilisation qui est faite des illustrations y rendent poreuses les frontières entre ces trois phases.

\section{Pèlerinages}

Le terme que Michelin a utilisé dès l'origine est celui de " pèlerinage ", ce en quoi il partage les objectifs d'autres guides contemporains (Ginisty / Alexandre 1916 ; Loez / Offenstadt : 212). Dans cette mesure, les illustrations insérées entre 1917 et 1937 pouvaient légitimer un esprit de ferveur auquel l'éditeur entendait répondre par l'authenticité et le réalisme optique qui se devaient de ressortir de ses gravures, de leur netteté et des légendes précisant l'année du cliché et la situation. Le code esthétique était celui du 
documentaire et chaque image témoignait, en somme, de la présence de reliques incrustées dans l'espace et laissant potentiellement la place aux émotions et au recueillement sécularisé ou religieux.

Cet esprit perdure jusqu'à nos jours : les lieux photographiés sont certes des buts touristiques mais aussi des endroits sanctuarisés : les illustrations, dont la majorité est dépourvue de personnages, incitent à un travail de deuil qui se perpétue au fil des générations. Ce qu'observe $A$. Hertzog (2012 : 28) comme un phénomène global actuel "Les valeurs de sacralité, de respect, le deuil, la commémoration du sacrifice n'ont pas disparu des registres discursifs " - peut s'appliquer à tous les Michelin des champs de bataille, bien que ce phénomène soit problématisable de façon différente selon les générations, comme nous le verrons plus loin.

Leurs illustrations servent d'emblée à un « tourisme de mémoire", cetermedemémoire, icisynonymedecommémoration, étant récurrent dans les paratextes, toutes dates confondues. Au départ, c'est l'entreprise qui gère ses publications comme si elles constituaient par elles-mêmes un mémorial dédié « À la mémoire des ouvriers et employés de Michelin morts pour la patrie ». De sorte que les gravures ont au fond une portée semblable à celle que l'on donnerait à la liste des noms de soldats morts. Cette interprétation est d'autant plus plausible que le foisonnement des photographies - il y en a en moyenne deux ou quatre à chaque double page - résulte d'un effort de donner chair au plus possible de toponymes, de la même façon que la description du trajet s'efforce de n'oublier aucun lieu. Si cette dédicace placée en exergue commémorait des vies sacrifiées, les illustrations concrétisent ensuite ce geste symbolique en montrant les lieux des combats. Pour enclencher le processus de mémorisation à partir de ce qui est matériel, les photographies qui sont placées en face du titre et plus tard sur la jaquette sont généralement une vue prise d'avion ou un panorama de façon à ce que le recul optique incite à sublimer le repère spatial de la destruction et de la mort.

\section{Tourisme d'histoire}

Michelin a également souligné dès l'origine que la fonction de ses guides était de servir aussi à un tourisme d'histoire. Ils commencent par un rappel des grands faits historiques concernant les villes, puis détaillent les faits militaires entre 1914 et 1918 - à partir de " l'invasion » de 1914 jusqu'à ce que le lieu « soit libéré ». Ils passent ensuite à la description de la ville quartier par quartier, faisant alterner description des monuments et photographies. Vient en troisième lieu la visite des lieux de batailles, découpée en demi-journées et ne concédant aucune échappatoire. S'il s'agit de zones rurales, comme dans les guides de 1917 sur les batailles de la Marne, les photographies se répartissent entre scènes de guerre et villages endommagés.

Tourisme d'histoire aussi du fait de la corrélation implicite entre le rappel du passé et les enjeux contemporains respectifs à propos de la valorisation des vestiges (Danchin 2015). Ainsi l'insistance placée sur l'incendie de la cathédrale de Reims peut-elle s'expliquer par la cristallisation de l'indignation nationale, par les vifs débats sur les modalités d'une restauration qui divisaient politiciens, militaires, inspecteurs des Monuments historiques, entre adeptes et détracteurs de la reconstruction, et ce sur toile de fond de la querelle entre historiens de l'art allemands et français quant à l'origine de l'art gothique (Gaehtgens 2018).

Enfin, tous - y compris les premières traductions en anglais - ont pour objectif de faire " comprendre » la guerre, les données factuelles, la logique selon laquelle les batailles se sont enchaînées et déroulées. Chaque ouvrage paru entre 1919 et 1921 s'est emparé d'un lieu, expose la configuration, s'appuie sur des cartes et des panoramiques ; en 2019 cette approche rationnelle de la géographie perdure et elle est globalisée puisque Michelin a choisi de regrouper tous les champs de bataille dans un seul et même guide.

Vu cette ambition de faire " comprendre ", ces guides sont, mutatis mutandis, des précurseurs : ils n'ont pas attendu la disparition des anciens combattants ou le rajeunissement du public pour présenter le résultat d'enquêtes sur le terrain. Photographies et passages explicatifs sont destinés dès la première heure à transmettre des informations de qualité. Leur historiographie est en quelque sorte semi-scientifique. Semi-scientifique seulement car fragmentée et dépourvue de notes si bien que Michelin propose certes des matériaux historiques, mais il s'efforce de ne pas ennuyer. Les documents des années 1917-1937 pouvaient intéresser un public qui se recrutait majoritairement dans les milieux aisés disposant de voitures, le guide de 2019 , plus aéré et coloré, est quant à lui susceptible d'attirer tout public.

\section{Tourisme et guerre ?}

La formule même de tourisme de guerre est un oxymore. Comment un loisir se greffe-t-il sur des lieux de combat et de mort ? Certains chercheurs décèlent une parenté entre le tourisme de guerre et le tourisme noir, macabre, le thanatotourisme, l'attirance de la mort. À l'opposé, certains sites internet d'aujourd'hui risquent d'anesthésier la douleur voire de banaliser la guerre, impression qui résulte des techniques infographiques.

Le risque inhérent à cet oxymore est écarté par Michelin. Outre la perspective historique, qui oblige à intellectualiser ce qui est montré, il pratique les chocs entre prises de vue. Par exemple la double page consacrée à Strasbourg en 2019 (p. 51) place deux photos en regard, à gauche «la villa Bismarck » (surnom d'une casemate située sur le front du Sundgau), prise en plongée et la montrant lovée dans un petit bois, et à droite le monument aux morts de Strasbourg, photographié en contre-plongée et si grand sur la page que c'est lui qui attire le regard. La juxtaposition des deux est 
sans équivoque : ni l'affliction de cette Pietà laïque de 1936' ni la visite du blockhaus de 1918 ne peuvent se réduire à une promenade distrayante. Les lieux photographiés sont donc certes des buts touristiques mais ils sont aussi des endroits sanctuarisés.

Tout en s'inscrivant dans des usages traditionnels du tourisme de guerre (Leonhard 2014 : 995), Michelin a mis en place des procédés innovants qui croisent pèlerinage, mémoire, histoire. Une des manifestations de ce croisement est le réemploi de ses photographies imprimées en 1919-1921 : étant reproduites et complétées au fil des rééditions, elles continuent à circuler, deviennent polysémiques et informent sur le glissement de la mémoire communicative à la mémoire culturelle de la Première Guerre.

\section{SORTIE DE GUERRE ET GUIDES ACCUSATEURS (1917-1921)}

Dans les éditions des années 1917-1921, les photos sont contemporaines de la publication, la mémoire communicative étant mise ici au service d'intérêts politiques et nationaux.

\section{Survivance de la propagande}

Les deux guides parus en 1917 sont placés sous le signe de la victoire, ce qui correspondait à la propagande souhaitée par les autorités militaires. La série qui paraît après l'armistice, dans les années 1919-1921, dissocie la partie relatant les combats et la visite, relativement traditionnelle, des localités ; les clichés datent d'avant guerre et sont ici ou là complétés pour montrer " l'état de désolation » dans lequel les troupes allemandes les ont laissées. La répartition des photographies y est encore marquée par le discours d'un État victorieux et accusateur, y compris pour les objets photographiés qui sont principalement des monuments. Puisque les clichés avaient été pris pendant la guerre, il importe de ne pas oublier que - derrière leur apparent réalisme - il y avait eu le contrôle des autorités militaires et que les motifs retenus étaient ceux qui n'avaient pas été entravés par la censure. Cela peut expliquer qu'aucun blessé ne soit montré, à de rares exception près pour Verdun, et que ce soient en revanche les plaies des monuments historiques, des habitations privées, des œuvres d'art, des paysages, qui soient exposées.

\section{Faire expier}

Le Michelin diffuse la haine de l'ennemi et la thèse d'une culpabilité allemande. Une photographie montre par exemple trois soldats allemands, surveillés par un Français, en train de déblayer les ruines dans une rue de Reims : or, même si c'est à très petite échelle par rapport à la diffusion que connaîtra, après la Seconde Guerre, l'image des Trümmerfrauen, cette

1 https://commons.wikimedia.org/wiki/File:Monument_morts_Place_R\%C3\%A9publique_Strasbourg_3.jpg [consulté le 19.05.2021]. scène de 1918 relève déjà de la symbolique de l'expiation à assumer.

Le discours victorieux et nationaliste est ouvertement affiché, il désigne ce qui serait à faire expier et dresse un inventaire des souffrances matérielles. Par exemple, dans le guide consacré à Reims en 1919, une photo prise depuis un aéroplane fait saisir l'étendue de la destruction de la ville et trouvera des équivalents dans les images de la Seconde Guerre. Elle avait d'ailleurs été déjà diffusée par la revue L'Illustration le 2 novembre 1918. L'incendie de la cathédrale de Reims est qualifié d'acte de "vandalisme », le commentateur - féru de connaissances historiques et littéraires - justifiant son accusation en rappelant que même les appels enfiévrés d'un Ernst Moritz Arndt n'auraient pas été suivis d'actes aussi barbares en 1813.

Quels que soient les exemplaires de cette époque, presque chaque village mentionné est assorti de la photo de son clocher. Le spectateur est périodiquement incité à voir en l'ennemi un iconoclaste tant il y a d'églises à demi-détruites : elles sont photographiées généralement de face si bien que le clocher endommagé et la plaie qu'il symbolise sont au centre. Dans le guide sur Verdun, un exemple modeste mais saisissant est le clocher de Charny, sur la rive gauche de la Meuse, qui semble sur le point de s'écrouler (Verdun 1929 : 104). Ce lieu de mémoire qu'est un clocher (Boutry 1997) est représentatif des mentalités au sortir de la Première Guerre : c'est à la fois pour leur connotation religieuse que les églises sont montrées et plus généralement pour des raisons sociales car elles restaient le centre, le lieu familier rythmant les heures et les périodes de la vie. III. 1.

\section{Considérations juridiques et exigences de réparations}

Si l'accent est mis sur l'indignation face aux destructions, alors qu'on ne voit pas ou peu les hommes en train de combattre, et si les photographies du patrimoine bâti sont aussi nombreuses que celles des paysages sacrifiés, c'est aussi que la culture touristique est instrumentalisée au service de la justification des exigences de réparations. Car les critères militaires et juridiques faisaient débat. À cet égard, Michelin occultait une réalité stratégique car, si les églises avaient servi pour la défense, leur destruction était autorisable aux termes de la convention de 1907. Ces débats sont à l'arrière-plan des guides de 1917/1919-1921. D'ailleurs, sur le tampon de l'exemplaire de Reims conservé à la Staatsbibliothek de Berlin figure la référence "Aufbauvertretung Paris » (Staatsbibliothek Berlin [50 MA 24019]) : ce guide entrait donc parmi les pièces transmises à Berlin, au Ministère de la Reconstruction, créé le 7 novembre 1919 qui gérait le paiement des réparations. ${ }^{2}$ Un autre exemple est une photographie insérée dans le Reims de 1919 montrant une forêt décimée et à propos de laquelle la légende précise que c'était l'endroit où une 2 Je remercie les services de la Staatsbibliothek pour avoir expliqué le contexte et
conseillé Dirk Hainbuch (2016), Das Reichsministerium für Wiederaufbau 1919 bis 1924 , Frankfurt a.M., Peter Lang. 


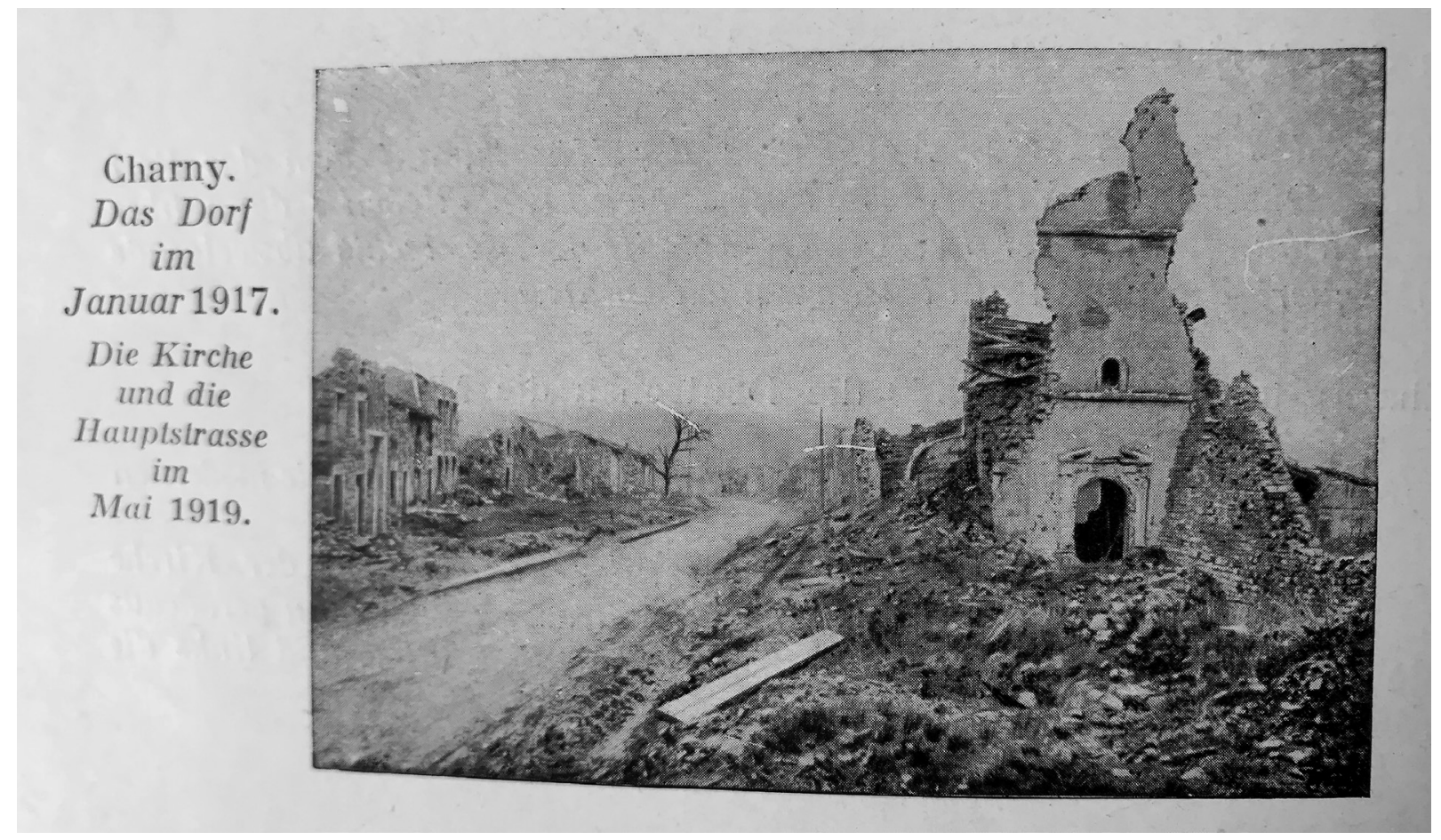

III. 1 Charny @ Michelin

scierie se fournissait précédemment en bois, ce qui donnait à entendre qu'une indemnisation serait à prévoir.

Ces considérations économiques et culturelles sont relayées par les photographies qui accentuent l'impression d'une blessure encore à vif telle que l'avait ressentie le journaliste $A$. Londres en 1914. Le scandale des destructions est dénoncé en opposant l'avant et l'après. À Reims, Verdun, Lille, on trouve en parallèle des photographies archivées par les monuments historiques avant la guerre et, à la page suivante, le même objet après le bombardement. Le visiteur est incité à reconstruire en imagination les parties qui ont entretemps disparu, par exemple la disparition de la toiture de la cathédrale de Reims (Reims 1919 : 28). L'imaginaire est alors inversé : les séquelles de la guerre sont reproduites dans leur factualité avec les gravats et le passé dans sa virtualité d'avant.

Michelin soutient certes la ligne française officielle qui réclamait le paiement des réparations, mais l'esthétique peut nous délivrer rétrospectivement un tout autre message. À preuve la photo qui ouvre le guide de Reims. "Confisquée à un prisonnier allemand » (Verdun 1919: 1), elle est un butin et un signe de vengeance : la ville a été reconquise par la France. Or, cette photographie présente un intérêt qui la sort de ce contexte belliqueux et qui pourrait en permuter la signification. Sa composition - une succession de trois plans mettant au centre le but espéré et lointain - captive l'attention ; et il n'est pas à exclure que le photographe allemand se soit inspiré de l'iconographie romantique et nostalgique d'un Caspar David Friedrich et de ses panoramas, par exemple de son tableau "Blick auf den Schmiedebergerkamm ». Le résultat est paradoxalement le message transnational d'une esthétique partageable. III. 2.
En résumé, dans ces guides de 1919-1921, la chose vue, le « référent » (Barthes 1980), reste les vestiges du récent affrontement militaire. Voyager est une manière de poursuivre la guerre autrement et d'afficher un patriotisme national. Les forts détruits sont si explicitement reliés aux opérations des régiments qu'ils ne sont ni traités en musées, comme ce sera le cas après 1927, ni ostensiblement pourvus d'un drapeau tricolore, comme en 2019. La priorité de cette série de guides était le devoir et l'ambition de transmettre aux futures générations ce qui resterait digne d'être connu et célébré, à savoir d'abord et avant tout le deuil collectif, celui de la nation. La mémoire collective s'y construit comme discours identitaire national : les motifs sélectionnés se complètent mutuellement de manière à englober les ruines du grand patrimoine historique aussi bien que celles des villages. Tout comme les forêts décapitées et les paysages crevassés, elles dégagent une impression d'accablement et exaltent les sentiments. La violence des combats est montrée par les effondrements et les souffrances par les béances du patrimoine bâti qui représente le martyre des populations. Mais la censure, cette fois intériorisée, continue à tabouiser la mort des hommes.

La culture touristique qui, en 1917, relevait encore du reportage et de l'effort de guerre, est instrumentalisée au service du politique et ces guides sont directifs au double sens du terme, spatial et intellectuel. L'accumulation des tableaux de désolation, en accord avec la technique du noir et blanc, sert non seulement à souder la nation au nom du patrimoine esthétique et de considérations juridiques mais aussi à transmettre le souvenir que l'identité française se serait bâtie autour du catholicisme et que, parmi les arguments culturels avancés durant la Guerre, les camps s'étaient respectivement affichés 


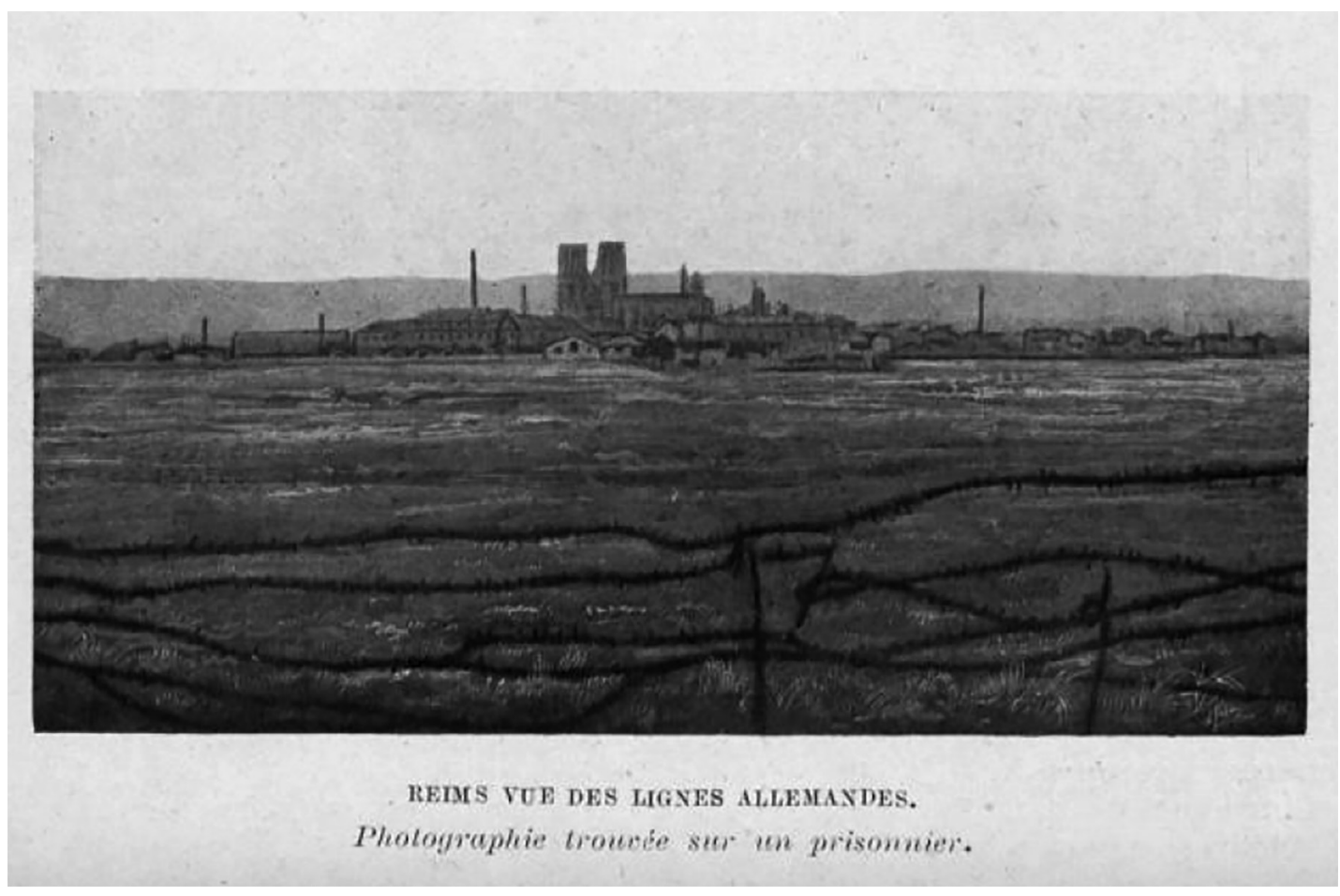

III. 2 Photographie trouvée sur un prisonnier $\odot$ Michelin

autour du catholicisme français et du protestantisme prussien (Pfeilschifter 1915). Ce qui signifie que la thématique des destructions se veut explicite et répond encore aux critères que la propagande avait utilisés pendant la Guerre même (Arendes 2016). La datation qui figure dans les légendes (1915, 1916, 1917) fait d'ailleurs constater que la continuité est délibérée.

\section{BRÉVIAIRES DE PĖLERINAGES À VERDUN (1925-1937)}

Entre les deux guerres, entre 1925 et 1937, le seul guide plusieurs fois réédité et augmenté par Michelin est celui de Verdun. Vu le décalage temporel, sa vocation peut se comparer à celle d'un bréviaire : comme en écho aux fêtes commémoratives qui y furent organisées (Prost 1997 : 1769-1771), passages rédigés et illustrations aidaient à tirer un enseignement de la guerre passée.

Étant donné ses rééditions et traductions, le guide de Verdun était probablement destiné en priorité aux descendants de soldats, aux vétérans souhaitant revisiter les lieux où ils se sont battus et aux historiens. Rien d'inattendu à la focalisation sur « la bataille qui résume à elle seule la Grande Guerre » (ibid.: 1761). Pour autant, quelle place prend ce guide pour aménager le passage progressif à la mémoire culturelle et transformer l'événement en symbole ? Par ailleurs, comme c'est le seul qui ait été traduit en allemand, les gravures - pensées en 1919 en termes de mémoire nationale - seraient-elles susceptibles de créer une mémoire transnationale ou au contraire de consolider les mémoires nationales respectives de chaque côté du Rhin ?

\section{Premières cicatrisations}

Les photographies qui dataient de 1916 et 1919 sont complétées par celles des monuments commémoratifs érigés entretemps pour officialiser le souvenir. Or, comme les illustrations remontent à une dizaine d'années, l'esthétique de ces anciennes prises de vue gagne à être regardée différemment et examinée en autonomie : en effet, ce ne sont plus les témoignages immédiats des plaies de la guerre, ce sont des vestiges qui, placés à proximité des vues récentes de nécropoles (cimetière américain, ossuaire de Douaumont, monuments aux morts, stèles), suggèrent que les blessures commencent à cicatriser.

Introduit par un encarté de l'œEuvre du Souvenir, chaque exemplaire de Verdun associe la visite du site à la célébration de diverses formes de commémorations, officielles dans les ossuaires ou plus individuelles sur le terrain. Si bien que, théoriquement, les photographies pouvaient aussi bien entretenir la haine de l'ennemi qu'au contraire consolider les thèses pacifistes. Kurt Tucholsky, sous le pseudonyme Ignaz Wrobel, a par exemple publié une émouvante relation de sa visite des lieux de bataille à proximité de Verdun (« Vor Verdun », Die Weltbühne, Berlin, 7-08-1924 : 218-222). Mais la mémoire de Michelin reste avant tout combattante et les photos servent l'historiographie militaire. Par exemple, chacune de ses rééditions commence par la vue aérienne dramatique intitulée "Sous les obus incendiaires, Verdun brûle (1916) ».

Se situant au carrefour de deux systèmes de signes, ces rééditions réemploient les anciennes photos liées à la sortie de guerre, ne montrent pas encore ce qui est reconstruit et ajoutent des clichés sur le quotidien des soldats pendant 
la guerre, ces derniers étant montrés dans des positions temps de pose obligeant - le plus souvent statiques. En outre, elles insèrent les nouveaux clichés des premiers bâtiments commémoratifs, ce qui met en place la représentation d'une solennité esthétisante, même s'il faudra attendre les années 2010 pour que les couleurs en accentuent l'effet comme sur la couverture du guide de 2019. III. 3 .

Il en va de même pour les cimetières militaires, dont les photos sont proportionnellement peu nombreuses puisque 42 " cimetières nationaux autour de Verdun " sont recensés à la fin du guide en français et 33 « cimetières allemands » à la fin du guide allemand.

Les perspectives illustrent le nombre de morts, les alignements de croix les suggérant quantifiables (les chiffres figurent dans les légendes) et étant moins aptes qu'en 2019 à esquisser l'incommensurable. Ou encore la vue du cimetière américain à Romagne, qui s'étale sur deux pages, obéit au cadrage strict d'un parallélépipède, la limite de cet enclos étant marquée par des arbres alignés au cordeau et par la hampe du drapeau, le tout étant surmonté en 1937 par la nécropole (Verdun 1937 : 128). III. 4.

Les vestiges qui figurent sur les anciennes photographies se transforment en objets de musée, quasiment figés hors du temps. D'ailleurs le commentaire écrit change car, en 1919, la fonction de beaucoup de sites photographiés restait stratégique et reconstituait les conditions du combat, alors qu'il s'ajoute en 1929 un processus d'esthétisation. Ce qui s'impose au regard est désormais moins l'accablement, que pouvaient jadis inspirer les accumulations de gravats, que la possible esthétique des ruines.

Par exemple, le guide de Verdun de 1919 avait montré l'observatoire allemand de Montfaucon attaqué et pris par les

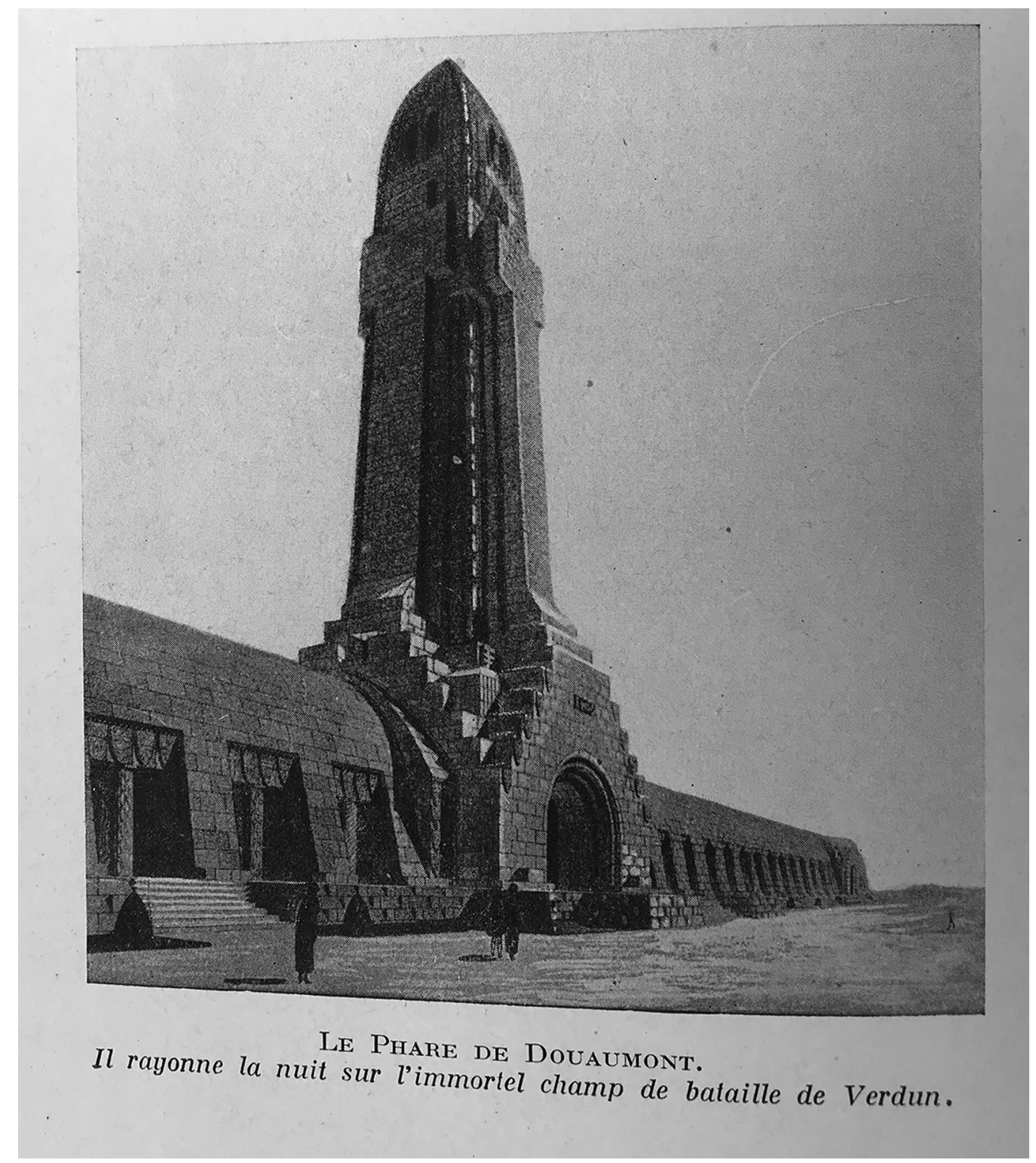




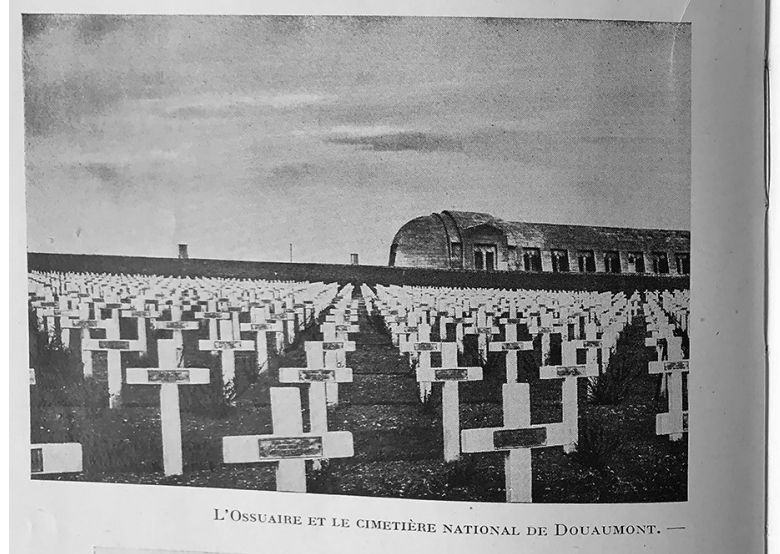

III. 4 Cimetière@Michelin

Américains : « L'observatoire allemand, d'où est pris, à travers le créneau, le panorama ci-dessus »; la photo de dessous était celle d'une arcature d'église restée debout. Ceux de 1929 et 1937 remplacent cette légende par « Au milieu des ruines de l'église, il fut construit avec les décombres de celle-ci » (ibid. : 127), ce qui introduit la dimension de la poésie des ruines. Et ce d'autant que la photo de l'arcature étant déplacée en bas de la page opposée, elle n'est plus en prise directe sur le combat.

Dans le contexte de l'entre-deux-guerres, un tel vestige constitue un atout pour un tourisme de nature mémorielle dorénavant en quête d'immatérialité. Cette photographie de l'arche est habilement structurée, organisée verticalement par les piliers, centrée autour de l'arc brisé, et des blocs jonchent le sol au premier plan, si bien, que, tout comme une fois encore un tableau romantique, par exemple celui de Carus Beschneite Tannen, elle peut inciter à méditer sur le temps et le sens de la guerre. Au demeurant c'est ici sans célébration d'une rédemption car cette arcature de 1937 ne s'ouvre pas vers un indéfini mystique : elle laisse au contraire apercevoir, tout au fond, le petit rectangle qu'est l'observatoire utilisé par les soldats. III. 5 et 6 .

\section{Effet de distanciation}

Ce genre de recherche esthétique peut accroître les émotions et en même temps créer un effet de distanciation. Les photographies des villages en sont un exemple. En 1919-21, rues désertes et clochers estropiés suggéraient un lourd silence et une absence totale de vie. Au contraire, depuis 1929, l'accent est mis sur le changement de civilisation consécutif à la guerre. Ainsi une nouvelle photo, celle d'une route traversant Esnes, village que les combats avaient rayé de la carte, inclut-elle un visiteur citadin chapeauté et lisant - peut-être un guide Michelin. En-dessous de cette mise en abyme figure la même rue avant-guerre, grouillant des activités quotidiennes, saisissant contraste qui anticipe le dépeuplement des campagnes et la sécularisation de la mémoire collective (Verdun 1937, 117). III. 7.

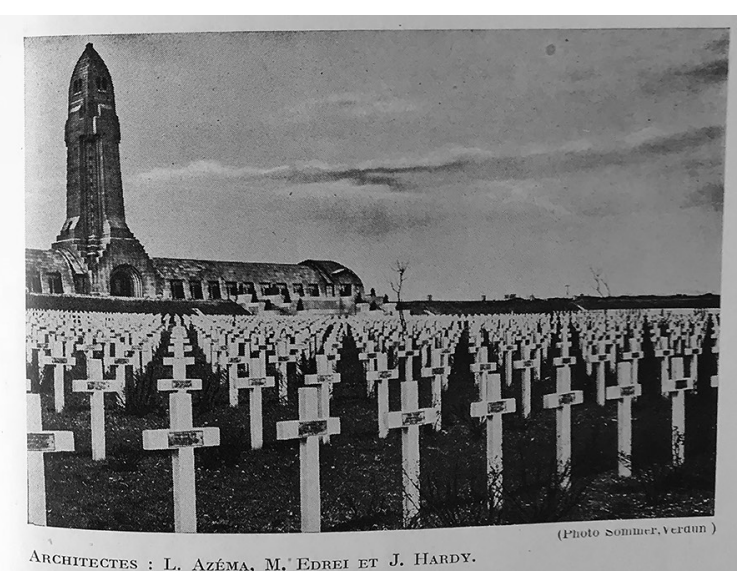

De réservoir de preuves, le guide de Verdun est délibérément devenu, à la fin des années 1920, un producteur de mémoire visuelle. Ce changement s'accompagne de l'insertion des reproductions de stèles et monuments aux morts avec la savante indication de leurs sculpteurs respectifs - utile aux générations futures. Et la légende de la photographie du " phare de Douaumont », qui était au futur dans le guide de 1929, passe au présent dans celui de 1937 : « II rayonne la nuit sur l'immortel champ de bataille de Verdun. »

\section{La traduction en allemand}

Le seul de ces Michelin qui ait été traduit en allemand est Verdun, Argonnen (1914-1918). L'avant-propos indique que c'est à la demande des touristes que cette version est éditée ; le but en serait de rappeler les " actes héroïques » qui se sont déroulés à Verdun et de rendre la visite « agréable et intéressante ». Ainsi transposées dans la culture de l'autre, les lignes de force des photographies restent le déroulement des batailles et les destructions associées à un pèlerinage qui s'inscrit dans l'émergence du mythe de Verdun.

Mais la consultation de ce guide donne l'impression que les mémoires n'étaient pas partagées : ce n'est certes plus la sortie de guerre qui y est représentée mais ce n'est pas pour autant une célébration de la paix. Non seulement le texte est traduit littéralement de sorte que la perspective reste française : les opérations de l'armée allemande restent vues comme étant celles de l'adversaire et les photographies sont identiques à la version en langue originale. Néanmoins la perspective allemande a induit à expurger tout le chapitre que le guide français avait consacré à Metz et à la « libération de Metz après l'armistice » (Verdun 1937 : 163-175) et la photographie d'un prisonnier allemand gisant à terre est supprimée - elle reste en revanche présente dans le guide français en 1937 (ibid. : 154).

Au total, renfermant les récentes photographies des mémoriaux et grands cimetières, les diverses versions du guide de Verdun de l'entre-deux guerres ont en commun de ne plus utiliser les ruines pour tabouiser les sacrifices en vies 


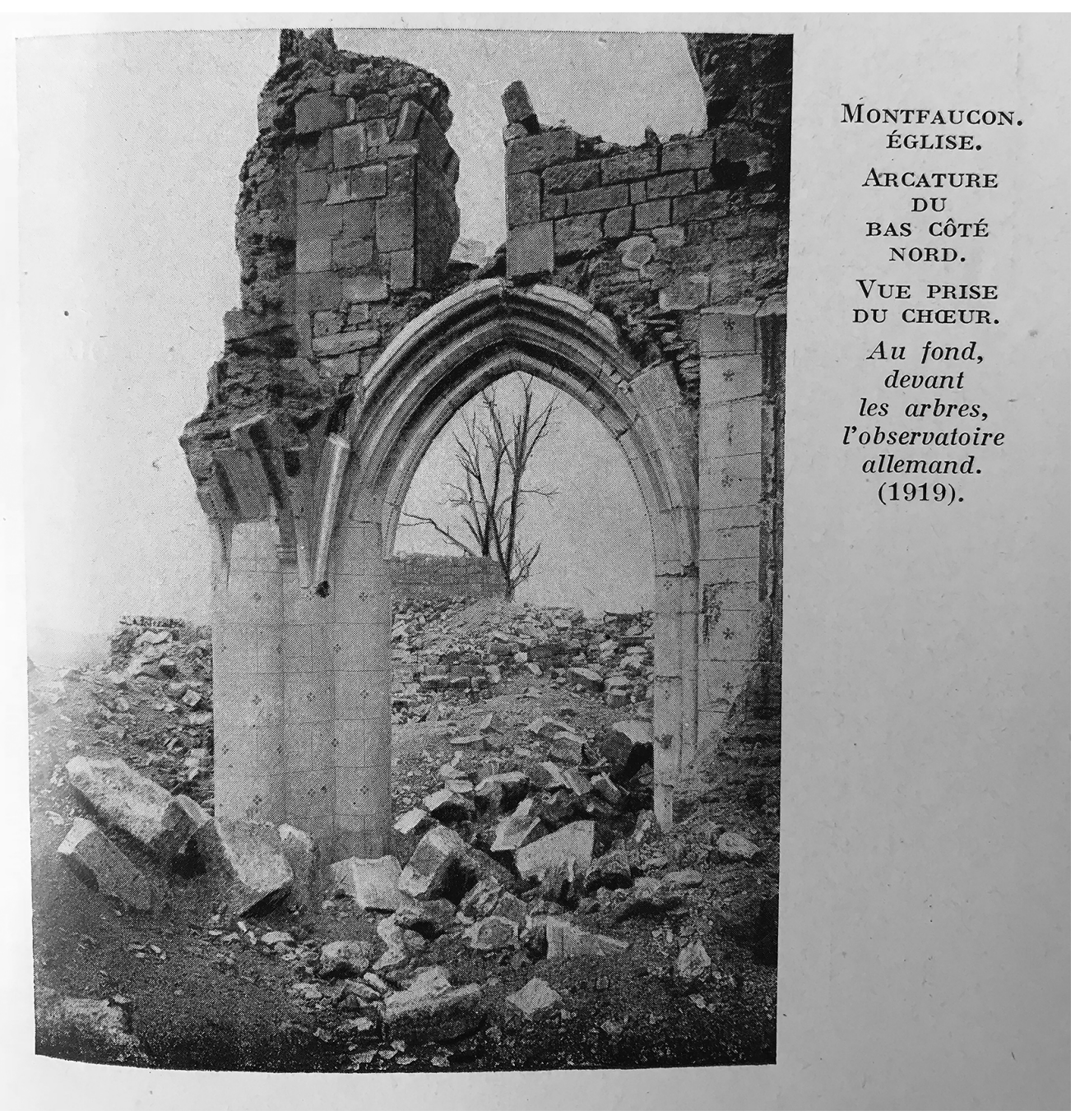

III. 5 Arcature@Michelin

humaines. La coprésence de clichés datant de 1916 et d'autres d'après 1921 a pour conséquence de transmettre ce qui, selon Michelin, resterait digne d'être connu et célébré dans la durée. Ce faisant, il s'en tient au culte du souvenir car les travaux de reconstruction des villes ne sont ni exploités ni problématisés.

De même l'internationalisation reste réduite. La dernière édition, celle de 1937, ne comporte pas du tout d'avant-propos. La mise en parallèle délibérée des photographies « Le général Pétain au milieu de ses troupes / General Pétain bei den Mannschaften » et "Le Kronprinz passant ses troupes en revue à Charleville / Der Kronprinz bei einer Truppenparade in Charleville » (Verdun 1937 et Verdun, Argonnen : 28-29) n'y est pas assortie de commentaire, leur opposition étant suffisamment explicite : le stéréotype de la discipline qui se dégage de la raideur du Kronprinz passant en revue les soldats allemands est opposé à l'image d'un Pétain bienveillant et paternaliste qui converse avec ses hommes.

Les alliés tiennent une place extrêmement réduite dans l'iconographie et chaque nation est enfermée dans son propre périmètre, comme si chacune avait à gérer séparément son hommage aux victimes et la formation de ses cicatrices. II en va tout autrement dans les guides parus de nos jours.

\section{L'ÈRE DE LA TRANSMISSION INTERGÉNÉRA- TIONNELLEETTRANSNATIONALE(2014-2019)}

Sa politique éditoriale, dans le cadre du centenaire de la Première Guerre, a conduit Michelin à mettre en œuvre deux procédés : la numérisation de quelques guides anciens, très utile mais ne rendant pas forcément compte de la répartition des photos sur deux pages ; la réédition augmentée de certains autres, retravaillés en utilisant les nouvelles technologies et en les ajustant aux pratiques culturelles des années 2010, c'est-à-dire en tenant compte de la culture de masse et de l'impact des films documentaires sur l'imaginaire collectif ; la frontière y est flagrante entre le passé de la guerre (restant en noir et blanc et centré sur les actions militaires) et les bâtisses (en couleurs) restaurées et aménagées en musées. 


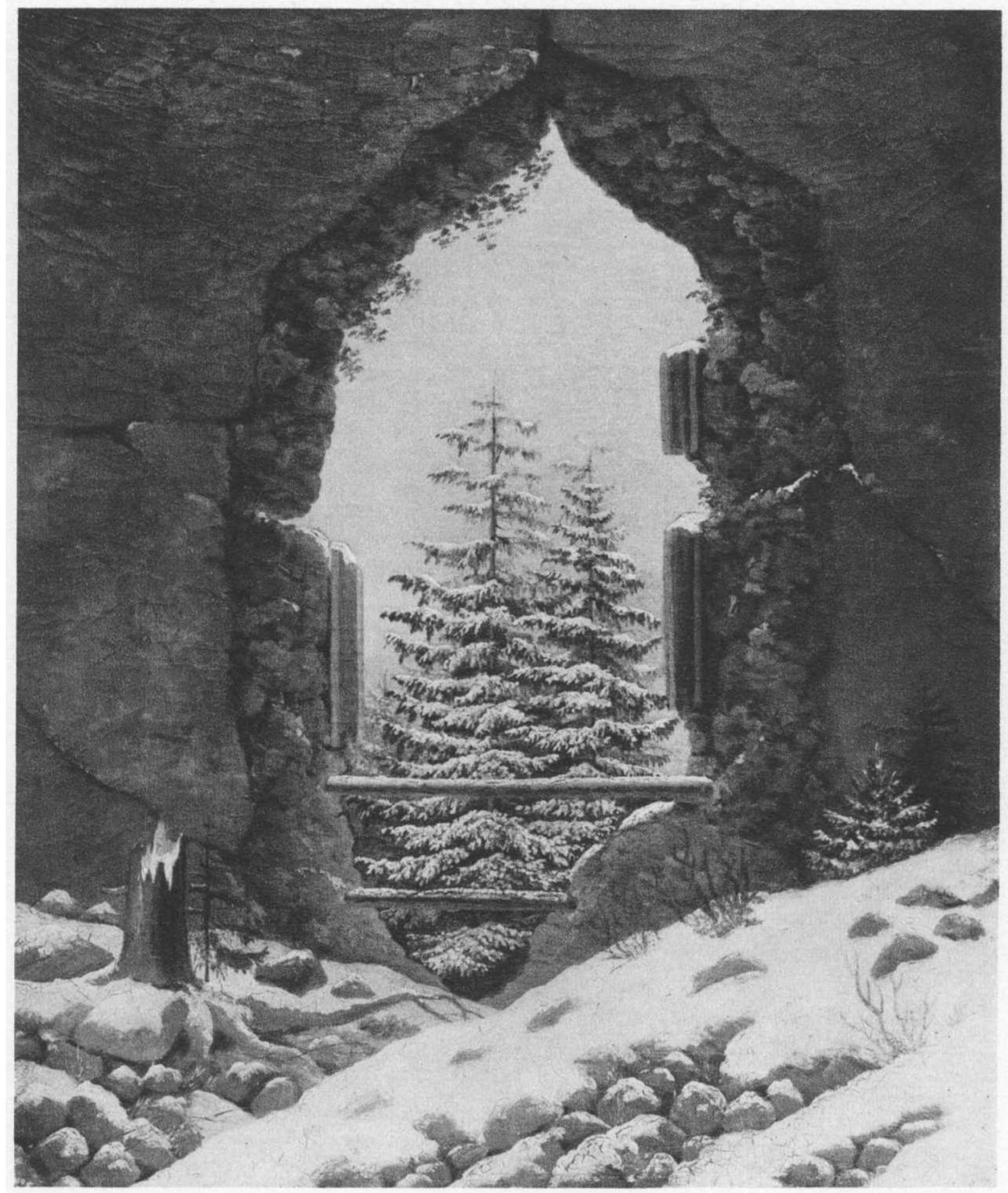

III. 6 Carus@commons wikimedia

\section{Le guide de 2019 pour « visiteurs de mémoire »}

La rupture avec l'ancienne iconographie porte aussi sur les représentations. C'est flagrant dans le guide sorti en 2019 car on y observe la transmutation contemporaine de la mémoire communicative en mémoire culturelle. Cet ouvrage regroupant les sites français et belges et l'espace n'étant plus fragmenté en volumes distincts, la notion de « champ de bataille » y est un terme générique qui ne correspond plus uniquement à un terme géographique et à une invitation au pèlerinage dans un périmètre donné, elle désigne avant tout un lieu de mémoire charriant plus immatériellement le souvenir des souffrances de la Première Guerre et les cicatrices qui se sont formées sur les plaies. Comme cela est écrit à propos du monument érigé pour les "victimes civiles 1914-1918 » à Cambrai, ce guide s'adresse aux « visiteurs de la mémoire » (Champs 2019 : 168). Le grand nombre des lieux répertoriés et des illustrations - en moyenne trois par page - reproduit sur papier le maillage serré des lieux de combat. Le plan global du guide reste identique aux précédents : il s'ouvre par une première partie chronologique rédigée par $F$. Cochet et se poursuit par la partie géographique.

La charge affective des motifs, en particulier des cimetières et des monuments aux morts, reste d'autant plus susceptible de se graver dans la mémoire visuelle du lecteur que, outre la recherche esthétique et la prégnance iconique (Assmann 2002), l'élargissement du « spectre géographique » à plusieurs champs de bataille permet « de réfléchir aux trajets des lieux et des mémoires » (Loez/Offenstadt : 31). Même si le spectre est limité dans ce guide à la France et la Belgique, les illustrations tiennent compte de I'historiographie actuelle qui aborde la Première Guerre « dans ce qu'elle a eu de mondial et à partir d'un point de vue transnational » (ibid.). C'est là un nouveau paradigme qui introduit une compatibilité entre l'ancrage patriotique et le respect des traditions militaires et nationales des autres pays. Les mémoires sont partagées. 


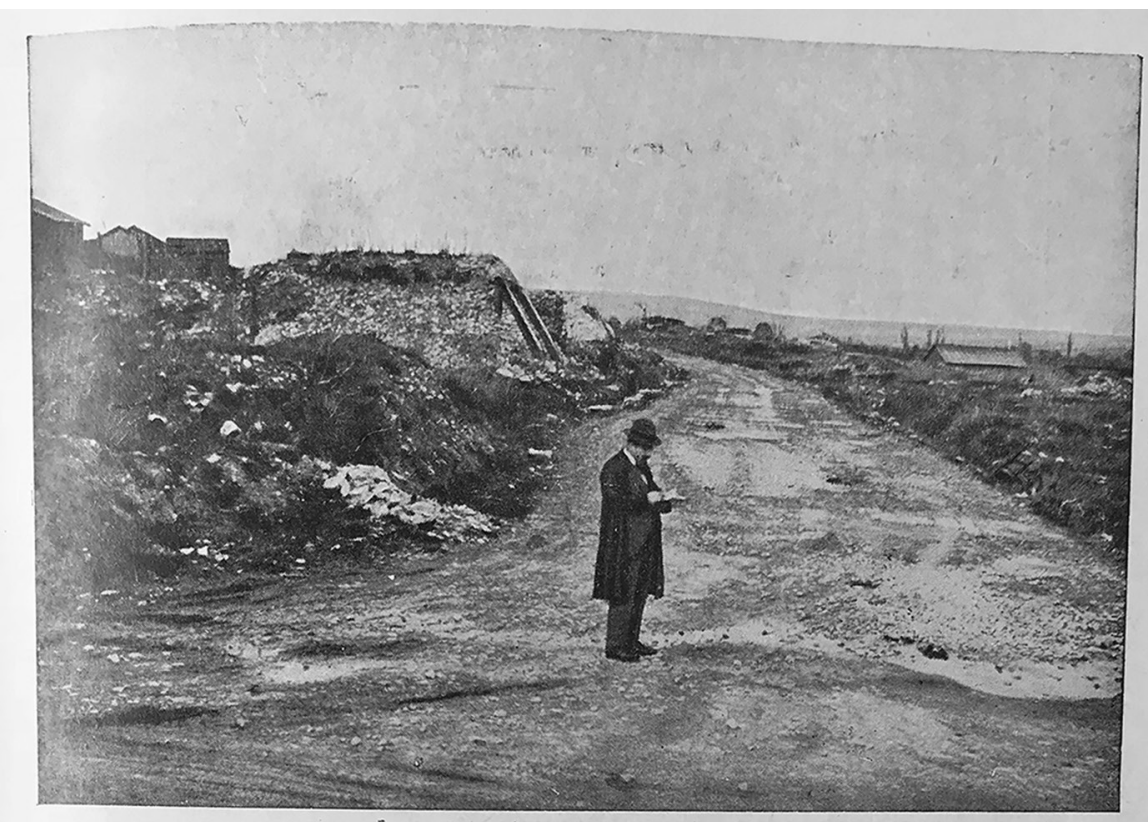

Esnes. - Die Strasse' nach Béthincourt im Mai 1921.

Nicht die linke Strasse einschlagen, da sie in einen sehr steilen Weg in schlechtem Zustand übergeht (Skizze S. 114).

Die zweite Strasse rechts führt in grosser Schleife zum Kreuzberg von Esnes, von dem aus man einen Ueberblick über die Schlachtfelder des linken Maasufers vom Toten Mann (Mort-Homme) bis zum Bourrus-Wald (BoisBourrus) hat. Am Kreuzberge zweigen zwei Strassen $a b$, die rechte nehmen. Diese Strasse geht zwischen Höhe 287 und der berühmten Höhe 304 durch.

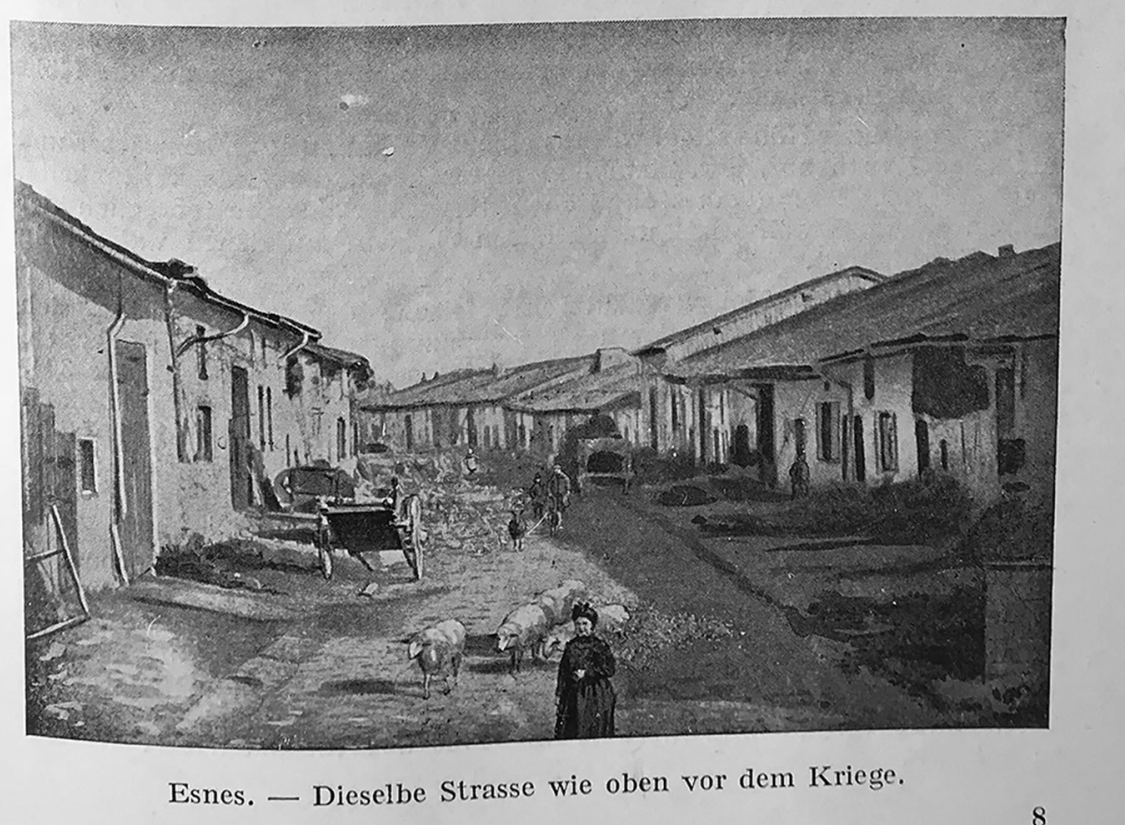

III. 7 Esnes@Michelin

C'est ainsi que les cimetières et les monuments aux morts dont les photographies jalonnent ce livre sont choisis de manière à la fois à présenter la diversité des décors et l'implication de plusieurs nations.

En outre, alors que les guides antérieurs ne s'étaient pas préoccupés de lier image et commentaire autrement que par la localisation, une interdépendance est établie entre les photographies et le texte de 2019 : les explications accordent une importance égale au descriptif de l'objet et à sa situation. Les détails du déroulement des batailles sont fortement résumés et les données culturelles (description des bâtiments visitables et mention des cérémonies qui s'y tiennent) l'emportent. La mémoire visuelle du lecteur est d'autant plus sollicitée que l'endroit désigné peut s'identifier 
rien qu'en observant l'illustration qui est placée en regard et précède la désignation du lieu répertorié, si bien que la halte prévue semble n'être justifiée que par la présence de ce qui est reproduit.

\section{Perspective muséographique}

Musées, nécropoles, cimetières, monuments aux morts stèles créent alors une autre cohérence que celle qui caractérisait les exemplaires précédents : la perspective est ici muséographique. Au lieu du foisonnement d'avant, la concentration se fait sur un seul motif significatif, les critères de sélection sont homogènes, et bien évidemment les techniques photographiques permettent de changer les angles de prise de vue.

Les objets privilégiés dans ce dernier guide Michelin officialisent le deuil. Cette fonction n'est plus assurée par des édifices religieux - même la cathédrale de Reims n'est plus représentée - mais par des bâtiments commémoratifs qui, mis en perspective de façon à leur conférer une taille monumentale, correspondent à une sécularisation du discours rituel traditionnel, transmettent sérénité, solennité, appel à la réflexion, et soulignent l'intention du guide de maintenir durablement cet attrait touristique.

Les forts, que 1919 montrait en piteux état, sont devenus des musées à visiter. Devant le fort de la Pompelle près de Reims flotte un drapeau tricolore, qui, moins que le rappel d'une victoire, indique que ce fort est géré par le ministère de la Défense. Les cimetières deviennent le signe d'un compromis mémoriel à cause de leur internationalisation, le guide veillant à ce que les nations impliquées dans la Guerre soient équitablement représentées. À cause aussi de leur ampleur : là où figuraient précédemment quelques croix plantées dans un pré, nous voyons maintenant des alignements innombrables, des perspectives infinies ; et la scénographie est travaillée de façon à ce que les lignes de fuite propulsent le regard vers le hors-champ. En outre, Michelin montre des cimetières déserts, sans touriste, à l'inverse de bien des photos qui circulent sur internet. C'est là une particularité de son style directif : en effet, face à un tel vide, chacun a le devoir d'être un acteur culturel, de venir se ressourcer et de méditer sur son appartenance identitaire.

\section{Résilience}

Ce guide de 2019 ne montre plus les répercussions matérielles de la Première Guerre mais en analyse les séquelles humaines et les constantes anthropologiques. C'est aussi à ce niveau que s'opèrent des glissements majeurs entre 1919 et 2019.

En 1919, les photos désignaient un passé qui ne passe pas. Les photos de 2019, elles, remémorent la guerre mais sans la représenter. Elles ont perdu leur fonction de preuves censées refléter le réel. Avec la part donnée à l'absence, au non-dit, le message devient atemporel,
« l'imaginaire » (Hertzog 2012) perdure et doit perdurer dans les mémoires. Les quelques tranchées et casemates, tout comme les ruines de deux abbayes cisterciennes, transmettent la consigne de les préserver pour rappeler ce qui s'est passé et pour témoigner au présent que la guerre est finie. À preuve aussi l'importance donnée à la couleur et à la végétation souvent printanière.

Ce passage du réel à " l'intelligible »-pour reprendre un terme de $\mathrm{N}$. Offenstadt - tient compte du besoin de commémorer sans prétendre être soi-même un héros. Il respecte en outre le besoin d'afficher les spécificités culturelles : la chapelle orthodoxe confère au cimetière russe un " parfum slave » (Champs 2019 : 93) et le dragon rouge, symbole du pays de Galles, évoque le régiment de Gallois morts ici (ibid. : 143). Outre son intention de " faire comprendre " le déroulement factuel des batailles, le Michelin de 2019 contribue, grâce au pouvoir symbolique de telles images, à une construction européenne et plurinationale de la mémoire culturelle, en la fondant à la fois sur la réflexion et les émotions, les deux interagissant si on se trouve sur les lieux de la guerre et même si le temps de la guerre est loin.

Les photographies de 2019 enseignent la résilience dans la mesure où elles peuvent avoir une fonction cathartique : selon celles de 1919, la punition de l'adversaire aurait été justifiée ; en revanche, celles de 1929-1937 et surtout 2019 remplacent l'affrontement par l'émotion esthétique et le recueillement. Les scènes de combats, quand elles sont insérées rétrospectivement, servent alors de contre-modèle et enrichissent le cadre réflexif sur l'éloignement de 14-18. Le réemploi de quelques photographies de combattants rappelle la réalité factuelle et l'hommage à rendre, les illustrations en couleur projettent dans le présent. Alors que les guides précédents racontaient la guerre en noir et blanc, celui de 2019 déborde de luminosité. Les images de destruction sont remplacées par des éléments structurés et stables, à telle enseigne que l'identité de chaque voyageur ou groupe de voyageurs peut se construire par compensation en constatant que des liens humains et sociaux avaient existé dans et par le déchirement de l'Europe de 14-18. Au demeurant, vus par les locaux, les territoires affichent leurs spécificités par le biais d'un patrimoine gardé, entretenu, utile aux intérêts économiques.

Le Michelin de 2019 décerne trois étoiles aux endroits qui scellent le triomphe de la reconstruction et qui favorisent l'idée que la matérialité d'aujourd'hui peut et doit faciliter la résilience, à l'inverse des amoncellements de gravats des anciens guides. Ainsi, au lieu de la Grand'Place d'Arras dévastée en 1917, le guide la montre aujourd'hui « restaurée à l'identique »; de Reims, il ne sélectionne que le monument aux morts ; et de la cathédrale d'Amiens le guide de 2019 reproduit seulement la plaque qui a été apposée en hommage aux Américains, quintessence d'une mémoire culturelle transnationale. Le référent n'est plus l'objet concret antérieur mais la représentation de sa signification telle qu'elle est produite par un artiste. 


\section{CONCLUSION}

Les guides de voyage sont des genres textuels qui se présentent comme essentiellement informatifs mais qui s'avèrent extrêmement directifs. Ils orientent le regard du visiteur et créent les modalités de son trajet, de ses observations et peut-être de ses impressions. Si le traitement des illustrations a permis d'examiner comment Michelin préparait la mémoire visuelle des visiteurs, sur le terrain et/ ou en pensée, une étude approfondie des textes explicatifs reste un des desiderata de la recherche. Elle mettrait en évidence, comme à propos des photogravures, un double mouvement : d'un côté les changements dus à l'ajustement aux pratiques mémorielles du temps et d'un autre côté la sédimentation de la gestion de l'espace, de la prise en compte des destinataires, de la sélection des objets photographiés, chaque génération se positionnant par rapport à ses prédécesseurs.

Les guides Michelin des champs de bataille ne sont pas des guides touristiques habituels : ils ont eu une importance éditoriale décisive, d'abord en s'adressant à une clientèle aisée, cultivée, puis de plus en plus élargie. Les endroits étant, dans l'immense majorité des cas, photographiés sans le moindre spectateur, cet anonymat transmettait un message plus globalement destiné à la communauté nationale au début, puis internationale par le biais des premières traductions, enfin nettement transnationale en 2019.

En 1917/1919-21, le photojournalisme des militaires ou de leurs supporters ont le monopole. C'étaient l'événement et la réalité matérielle de la destruction qui s'imposaient. Durant l'entre-deux-guerres ce monopole des photographies militaires se renforce mais il se complexifie puisqu'il se concentre sur Verdun, à la fois référent concret et mythe émergent. Avec le tournant pris par le guide de 2019, le visiteur ne peut plus avoir une connaissance objectivante de la Première Guerre, l'expérience qui lui est proposée n'est ni exaltation nationaliste, ni distraction délassante, ni angoisse de la mort : Michelin décrypte les symboles sélectionnés et relève le défi de concilier l'intérêt de tous et l'empathie de chacun.

En définitive, les illustrations des guides Michelin ne sont pas des instantanés figés proposant seulement un état des lieux à visiter à un moment donné : elles sont le signe de charnières mémorielles. Les premières traduisaient le climat de la sortie de guerre et la difficile transition entre guerre et paix. La deuxième série oscille entre souvenir des témoins et mémoire culturelle. La troisième change les paradigmes et prend le présent comme point de départ pour commémorer le passé et le préserver symboliquement dans le futur.

\section{BIBLIOGRAPHIE DES OUVRAGES CITÉS}

Guides Michelin cités :

(1917) L'Ourcq : Chantilly - Senlis - Meaux, 1, Paris, Berger-Levrault. (1919) La Deuxième bataille de la Marne, Clermont-Ferrand, Michelin. (1919) Reims et les batailles pour Reims, ibid.

(1919) Verdun Argonne Metz 1914-1918, ibid.

(1919) Lille avant et pendant la guerre, ibid.

(1919) Arras et les batailles d'Artois, ibid.

(1929) Verdun - Argonnen (1914-1918). Ein Führer, ein Panorama, eine geschichtliche Darstellung, ibid.

(1937) Verdun Argonne Metz 1914-1918, ibid.

(2019) Champs de bataille de la Première Guerre mondiale en France et en Belgique, ibid.

Arendes, Cord (2016), « Wirklichkeitsbilder? Fotografie und die Erinnerungen an den Ersten Weltkrieg », in Loureda, Óscar, Der Erste Weltkrieg und die Folgen, Winter, Heidelberg, 25-49.

Assmann, Aleida (2002), « Das Bildgedächtnis der Kunst, seine Medien und Institutionen », in Huber, Hans Dieter / Lockemann, Bettina / Scheibel, Michal (éd.), Bild, Medien, Wissen ; visuelle Kompetenz im Medienzeitalter, München, kopaed, 209-222.

Barthes, Roland (1980), La Chambre claire. Note sur la photographie, Paris, Gallimard.

Becker, Annette (2014), "Visual essay », in Winter, Jay (éd.), The Cambridge History of the First World War, Cambridge University Press, 640-643.

Beaupré, Nicolas (2014), « La Grande Guerre : du témoin à l'historien, de la mémoire à l'histoire ? », in Témoigner. Entre histoire et mémoire, 118, 54-60.
Boutry, Philippe (1997), « Le clocher », in Nora, Pierre (éd.), Les lieux de mémoire III, Paris Gallimard, 3081-3107.

Brandt, Suzanne (1994), "Le voyage aux champs de bataille ", in Vingtième siècle, 41, 18-22.

Cochet, François (éd.) (2006), 1916-2006, Verdun sous le regard du monde : actes du colloque tenu à Verdun les 23 et 24 février 2006, Paris, 14-18.

Danchin, Emmanuelle (2015), Le temps des ruines, 1914-1921, PUR, Rennes.

Düllo, Thomas (2011), Kulturals Transformation: Eine Kulturwissenschaft des Performativen und des Crossover, Bielefeld, Transcript.

Gaehtgens, Thomas (2018), Die brennende Kathedrale. Eine Geschichte aus dem Ersten Weltkrieg, München, Beck.

Ginisty, Paul / Alexandre, Arsène, Le Livre du souvenir, guide du voyageur dans la France envahie en 1914, Paris, Flammarion.

Harp, Stephen L. (2001), Marketing Michelin. Advertising and Cultural Identity in Twentieth Century France, Baltimore/London, John Hopkins University Press.

Hertzog, Anne (2012), « Tourisme de mémoire et imaginaire touristique des champs de bataille », Via, 1 [https://doi.org/10.4000/ viatourism.1276].

Heymel, Charlotte (2007), Touristen an der Front: das Kriegserlebnis 1914 - 1918 als Reiseerfahrung in zeitgenössischen Reiseberichten, Berlin, Lit.

Jalabert, Laurent / Puton, Jean-Pierre (2014), « La photographie de la Grande Guerre, affirmation d'un témoignage patrimonial », in In Situ. Revue des patrimoines 23 [https://journals.openedition. org/insitu/10992]. 
Krumeich, Gerd (1994), « Kriegsfotografie zwischen Erleben und Propaganda. Verdun und die Somme in deutschen und französischen Fotografien des Ersten Weltkriegs ", in Daniel, Ute/ Siemann, Wolfram (Hrsg.): Propaganda. Meinungskampf, Verführung und politische Sinnstiftung 1789-1989, Fischer, Frankfurt a. M., 117-132.

Leonhard, Jörn (2014), Die Büchse der Pandora. Geschichte des Ersten Weltkrieges, München, Beck.

Loez, André / Offenstadt, Nicolas (2013), La Grande Guerre. Carnet du centenaire, Paris, Albin Michel.

Moulin-Bourret, Annie (1997), Guerre et industrie. Clermont-Ferrand, 1912-1922 : la victoire du pneu, Clermont-Ferrand : Institut d'études du Massif Central.

Offenstadt N. (2010), La Grande Guerre aujourd'hui, Paris, Odile Jacob.

Offenstadt N. (dir.) (2004), Le Chemin des Dames, de l'événement à la mémoire, Paris, Stock.

Paul, Gerhard (2009), Das Jahrhundert der Bilder 1900-1949, Göttingen, Vandenhoeck \& Ruprecht.
Pfeilschifter, Georg (1915), Deutsche Kultur, Katholizismus und Weltkrieg, eine Abwehr des Buches'La Guerre allemande et le catholicisme', Freiburg, Herder.

Prost, Antoine (1997), "Verdun », in Nora, Pierre (o.c.), Lieux de mémoire II, 1755-1780.

Prost, Antoine / Krumeich, Gerd (2015), Verdun 1916 : une histoire franco-allemande de la bataille, Paris, Tallandier.

Riederer, Günter (2008), «Einleitung. Ein Krieg wird besichtigt - Die Wahrnehmung des Ersten Weltkriegs im Tagebuch von Harry Graf Kessler (1914-1916) » in Harry Graf Kessler, Das Tagebuch, Bd. 5, 1914-1916, Stuttgart, Klett-Cotta, 67-69.

Rohde, Horst / Ostrovsky, Robert (1992), Militärgeschichtlicher Reiseführer. Verdun, Herford/Bonn, Mittler.

Salzmann, Jean-Pierre (2006), « La bataille de Verdun, un but touristique. Les guides Michelin des champs de bataille de guerre de 1914-1918 », in Cochet (o.c.), 327-349.

Stoellger, Philipp / Gutjahr, Marco (2014), Visuelles Wissen: ikonische Prägnanz und Deutungsmacht, Würzburg, Königshausen \& Neumann. 